\title{
Cultural Variations in the Effect of Interview Privacy and the Need for Social Conformity on Reporting Sensitive Information
}

\author{
Zeina M. Mneimneh ${ }^{1}$, Roger Tourangeau ${ }^{2}$, Beth-Ellen Pennell ${ }^{1}$, Steven G. Heeringa ${ }^{1}$, and \\ Michael R. Elliott ${ }^{3}$
}

\begin{abstract}
Privacy is an important feature of the interview interaction mainly due to its potential effect on reporting information, especially sensitive information. Here we examine the effect of thirdparty presence on reporting both sensitive and relatively neutral outcomes. We investigate whether the effect of third-party presence on reporting sensitive information is moderated by the respondent's need for social conformity and the respondent's country of residence. Three types of outcomes are investigated: behavioral, attitudinal, and relatively neutral health events. Using data from 22,070 interviews and nine countries in the cross-national World Mental Health Survey Initiative, we fit multilevel logistic regression to study reporting effects on questions about suicidal behavior and marital ratings, and contrast these with questions about having high blood pressure, asthma, or arthritis. We find that there is an effect of thirdparty presence on reporting sensitive information and no effect on reporting of neutral information. Further, the effect of the interview privacy setting on reporting sensitive information is moderated by the need for social conformity and the cultural setting.
\end{abstract}

Key words: Privacy; cultural variability; interview variability.

\section{Introduction}

Many studies instruct interviewers to conduct their interviews in a private setting (with no third party present). The rationale is that the presence of a third party during the interview might interfere with the response process, possibly causing respondents to misreport information (especially that of a sensitive nature) or to rely on others present during the interview for answers to knowledge questions.

However, establishing interview privacy might not always be feasible, even when the study protocol calls for it. Most studies in different countries that report whether interviews were carried out in private reported rates of third-party presence higher than 35 percent (Mneimneh 2012).

The relatively common presence of a third party during the interview has led researchers to examine the effect of third-person presence on reporting answers to survey questions. In 1997, Aquilino proposed a framework that describes three factors affecting the size and

1 University of Michigan - Survey Research Center, Ann Arbor, MI 48106-1248, U.S.A. Emails: zeinam@umich.edu, bpennell@umich.edu and sheering@umich.edu

${ }^{2}$ Westat, 1600 Research Boulevard, Rockville, MD 20850, U.S.A. Email: RogerTourangeau@westat.com

3 University of Michigan - School of Public Health, Dept. of Biost., M4041 SPH II, 1420 Washington Heights, Ann Arbor, MI 48105, U.S.A. Email: mrelliot@umich.edu 
direction of bystanders' effect on reporting sensitive information ("bystander" and "third person" are used interchangeably in this article). The first is whether the survey question asks about factual or subjective information. The second is whether the bystander knows the factual information requested. The third is the perceived likelihood that the respondent might experience negative consequences by revealing new and unwelcome information to the bystander. If the third party already knows the information to be reported, then their presence might not have an effect, or it might even lead to more truthful reporting. If the third party does not know the information requested, and the respondent perceives a high likelihood of negative consequences by revealing this information, then the presence of a third party might lead to misreporting. The last two factors are related to the type of relationship that exists between the third party and the respondent. The relationship must be significant to the respondent, and the respondent's answer must bear directly on the relationship for the respondents to change their answers in order to convey a more desirable image in the presence of the third person (Aquilino 1997; Pollner and Adams 1997). This has led a number of researchers to investigate the effect of specific types of relationships on reporting answers to sensitive factual and attitude questions.

The most commonly studied types of relationship between the third party and the respondent are parental and spousal relationships. Several studies have investigated the effect of parent or spouse presence during the interview on reporting sensitive factual information. The effect of parent presence on reporting substance use among youth and young adults is consistent. Youth and young adults interviewed in the presence of their parents were less likely to report substance use (Aquilino 1997; Aquilino et al. 2000; Gfroerer 1985; Hoyt and Chaloupka 1994; Moskowitz 2004). In a meta-analysis conducted by Tourangeau and Yan (2007), the authors concluded that parental presence significantly reduced reporting of socially undesirable information.

The effect of spouse presence has been less consistent. In one of the studies conducted by Aquilino (1997) among married couples less than 34 years old, spouse presence had no effect on reporting substance use. However, in another sample, Aquilino et al. (2000) found higher rates of reported substance use among respondents (less than 45 years old) who were interviewed in the presence of their spouse. Casterline and Chidambaram (1984) studied the effect of third-party presence on reporting contraceptive use in several countries in Latin America, the Caribbean, Asia, and Africa. The authors found that husband presence during the interview reduced the odds of reporting contraceptive use. Pollner and Adams (1994) found that spouse presence reduced the reporting of depression symptoms among adult respondents residing in Los Angeles, but it did not have an effect on reporting other mental-health symptoms.

The effect of third-party presence on reporting subjective information has also been mixed. In India, youth and young adults (15-29 years old) interviewed in the presence of their parents reported more positive attitudes toward family (Podmore et al. 1975). In the United States, respondents interviewed in the presence of their spouse reported a better quality of marital life (Aquilino 1993). On the other hand, Anderson and Silver (1987) found that partner presence had no effect on agreement between emigrant Soviet couples when asked about their satisfaction with housing and standard of living. Pollner and Adams (1997) and Smith (1997) also reported that spouse presence had no effect on respondents' attitudes toward spouse support and satisfaction with household 
arrangements and subjective questions on marriage and gender differences, respectively. Pollner and Adams (1997) concluded that the inconsistency of findings and the ambiguity surrounding the interview conditions of some studies indicated that a conclusive judgment about third-party effects on reporting was not possible and needed further investigation.

Several factors might have contributed to the inconsistency of these findings, including interviewer differences in reporting privacy measures and a failure to take into account respondent characteristics that are associated with both reporting of sensitive information and establishing a private interview. These respondent characteristics could moderate the effect of third-party presence on reporting.

The first factor, interviewer differences, is important since interviewers are relied on to request, achieve, and report on interview privacy. We have shown that betweeninterviewer variation in reporting privacy measures is large (even larger than betweencountry differences) and could possibly vary from one study to another depending on interviewer training protocols and the population studied (Mneimneh 2012). To date, none of the studies investigating the effect of third-party presence on reporting controlled for interviewer variation in interview privacy.

The second factor involves respondent characteristics that could moderate the effect of third-party presence on reporting sensitive information. These include respondents' need for social conformity and their cultural background. Respondent's need for social conformity is driven by the respondent's motivations and desire to obtain social approval from others (Cialdini and Goldstein 2004) and minimize possible negative evaluation by others (Johnson and van de Vijver 2003). Such conformity motivations could be activated and strengthened depending on contextual stimuli, such as the perception of threats to fitting in socially and the lack of anonymity of the interaction (Cialdini and Goldstein 2004). Therefore, the presence of others during the interview may intensify such motivations already held by certain respondents. Thus respondents with activated conformity motivations might be more likely to misreport sensitive information than those with low conformity motivations. To our knowledge, only one study controlled for a similar conformity construct when investigating the effect of third-party presence on reporting (Moskowitz 2004). The author did not investigate the possible moderating effect of the respondent's need for social conformity on interview privacy and reporting, however.

Respondents' cultural background is another characteristic associated with both interview privacy and social-desirability motivation. The respondent's cultural background could be defined in several ways. Throughout this article the term cultural background refers to the country where the respondent resides or originates from. Respondents who reside in collectivist and lower-income countries were more likely to be interviewed in the presence of a third party than those residing in individualistic and high-income countries, respectively (Mneimneh 2012). This is consistent with how collectivist cultures are structured. In collectivist cultures, the self is defined in terms of relationships with others. To maintain harmony and interdependence in such cultures, close attention is given to others in the social context, especially if they belong to the ingroup circle-the individual's family and friends and others concerned with his or her welfare (Smith et al. 2006; Triandis 1995). Hofstede et al. (2010) discuss how a 
collectivist culture considers it normal for a member of one's in-group to invade one's privacy at any time. This stands in contrast to individualistic cultures, where ties between individuals are loose and the primary concern is independence (Hofstede et al. 2010; Triandis 1989). Cultural differences due to masculinity also seem to affect the presence of partners during the interview. Countries high in masculinity have distinct gender roles and are more focused on achievement and material success. The higher the masculinity of the country, the less likely a partner will be around during the interview (Mneimneh 2012).

The relationship between the interview privacy setting and wealth seems to be driven by the country's level of individualism and masculinity (Mneimneh 2012). As a country's wealth increases, its citizens give more attention to self-expression and personal choice. Moreover, as wealth increases, resources and commodities become more available, allowing citizens to become more independent rather than interdependent (Hofstede et al. 2010; Smith et al. 2006).

Respondents' cultural background is also associated with socially desirable reporting behavior. Most published literature has focused on the collectivism dimension and found it to be positively associated with general measures of social desirability (Bernardi 2006; Bond and Smith 1996; Triandis 1995) or specific components of social desirability, namely impression management (Lalwani et al. 2006). Triandis (1995, cited in Johnson and van de Vijver 2003), discussed how honesty in interactions with strangers is valued more in individualist societies, while saving face is more salient in collectivist societies. In collectivist societies, an individual's loss of face can also cause a loss of face for the group they belong to. Thus members that belong to the same ingroup have a shared interest in avoiding any loss of face to maintain in-group harmony (Ting-Toomey 1999).

Other cultural dimensions found to be associated with social desirability are a country's level of uncertainty avoidance (Bernardi 2006) and wealth (van Hemert et al. 2002). The association between social desirability and these two cultural dimensions, however, work in opposite directions: whereas a country's level of uncertainty avoidance shows a positive association with social-desirability motivation, a country's level of wealth exhibits a negative association. Neither masculinity nor power-distance dimensions have been found to be associated with social desirability (Bernardi 2006).

In summary, the perception of one's role and status vis-à-vis the roles and statuses of others in any social interaction is unconsciously guided by one's cultural background (Hofstede et al. 2010); as a result, respondents in certain cultural settings - for example, those living in collectivist (vs. individualistic) and lower-income (vs. high-income) societies - may be more concerned about how they appear to others present during an interview, leading them to misreport information. These hypothesized associations are guided by the previously demonstrated relationship between a country's level of collectivism and wealth and social-desirability bias. In this article, we specifically focus on the cultural factors that have been shown to be associated with both interview privacy and social desirability (a country's wealth and its level of individualism).

To understand the moderating effect of respondents' need for social conformity and the cultural setting on the third-party presence and reporting of sensitive behaviors (suicidal behavior) and attitudes (marital rating), we tested the following hypotheses: 


\section{Suicidal behavior:}

Hypothesis 1: There is an interaction between the respondent's need for social conformity and third-party presence. Reporting differences due to third-party presence are larger among respondents with a high need for social conformity.

Hypothesis 2: There is an interaction between the country's level of individualism and third-party presence. The effect of third-party presence on reporting suicidal behavior is reduced as the country's level of individualism increases.

Hypothesis 3: There is an interaction between the country's level of wealth and thirdparty presence. The effect of third-party presence on reporting suicidal behavior is accentuated in countries with a middle and low GNI per capita (compared to countries with a high GNI per capita).

\section{Marital rating:}

Hypothesis 4: There is an interaction between the respondent's need for social conformity and partner presence. Reporting differences due to partner presence are larger among respondents with a high need for social conformity.

Hypothesis 5: There is an interaction between the country's level of wealth and partner presence. The effect of partner presence on marital ratings is accentuated in countries with a middle and low GNI per capita (as compared to countries with a high GNI per capita).

For comparative purposes, we also tested the effect of third-party presence on relatively neutral health measures (having high blood pressure, arthritis, or asthma) using the following hypotheses:

\section{Physical chronic conditions outcome.}

Hypothesis 6: Third-party presence is not significantly related to the likelihood of reporting any of the physical chronic conditions.

Hypothesis 7: There is no significant interaction between the respondent's need for social conformity and third-party presence on reporting any of the physical chronic conditions.

Hypothesis 8: Neither the country's level of individualism nor the country's level of wealth significantly moderates the effect of third-party presence on reporting any of the physical chronic conditions.

From a theoretical point of view, testing for the above hypotheses will help survey researchers to better understand the effects of interview privacy on reporting sensitive information and shed the light on some of the inconsistencies in the literature. It emphasizes the importance of respondents' cultural and individual-level characteristics in moderating such effects and the need to account for them in future investigations. From a practical point of view, this research highlights the need to design well-defined interviewer privacy observations to capture the dynamics of the interview interaction and further investigate its effects on different types of questions. 


\section{Methods}

Data from the World Mental Health (WMH) Survey Initiative were used to address these research questions.

The survey design, implementation, and data processing across all participating $\mathrm{WMH}$ countries were coordinated by two central organizations. All participating countries were required to follow a standard survey protocol that includes a probability-sample design, a fixed minimum sample size, a shared core instrument, a specific translation protocol, a set of quality-control measures, and specific interviewer-training protocols. However, countries were allowed to adapt certain features, including computerization of the interview, the contact protocol, respondent incentives, and the field-team structure. This mix of centralization and local control allowed establishing a survey protocol adapted to local conditions while maintaining comparability of the data collected (Pennell et al. 2010).

\subsection{Sample Designs for the WMH Surveys}

In the first twenty-four countries that completed the WMH surveys, nine - Brazil, Bulgaria, Japan, Lebanon, Mexico, Nigeria, the People's Republic of China, Romania, and the United States - collected data on the respondent's social-conformity motivations and interview privacy. The analyses focused on these nine countries.

All WMH surveys targeted the adult population and most of them featured nationally representative probability samples of individuals in households. One (Mexico) was representative of urban areas, one (Nigeria) of selected states, and four (Brazil, India, Japan, and the People's Republic of China) of selected metropolitan areas. Detailed information on the survey sample design is published elsewhere (Heeringa et al. 2008).

To reduce interview length, the WMH interviews were designed to be administered in two parts: Part 1 included core questionnaire sections and Part 2 included noncore sections. All respondents completed Part 1; Part 2 was administered to a subsample of Part 1 respondents. The current analyses focused on Part 2 respondents as the scale measuring the respondent's need for social conformity and the majority of key outcomes were collected only in Part 2. Table 1 presents the number of Part 2 interviews completed in each country and the number of field interviewers.

\subsection{Questionnaire}

The WHO Composite International Diagnostic Interview (CIDI) Version 3.0 was used in all WMH surveys. The CIDI 3.0 is a fully structured interview that generates diagnoses for a wide range of mental-health disorders. It also collects information on treatment, disability, and physical chronic conditions. Detailed questions on social and family life, employment history, finances, and childhood experiences are also included. The questionnaire was translated into each country's local language following the WHO translation guidelines (Harkness et al. 2008; Kessler et al. 2004; Kessler and Üstün 2004).

\subsection{Questionnaire Administration}

In all countries, trained interviewers conducted face-to-face interviews using either paperand-pencil interviewing (PAPI) or computer-assisted personal interviewing (CAPI) 


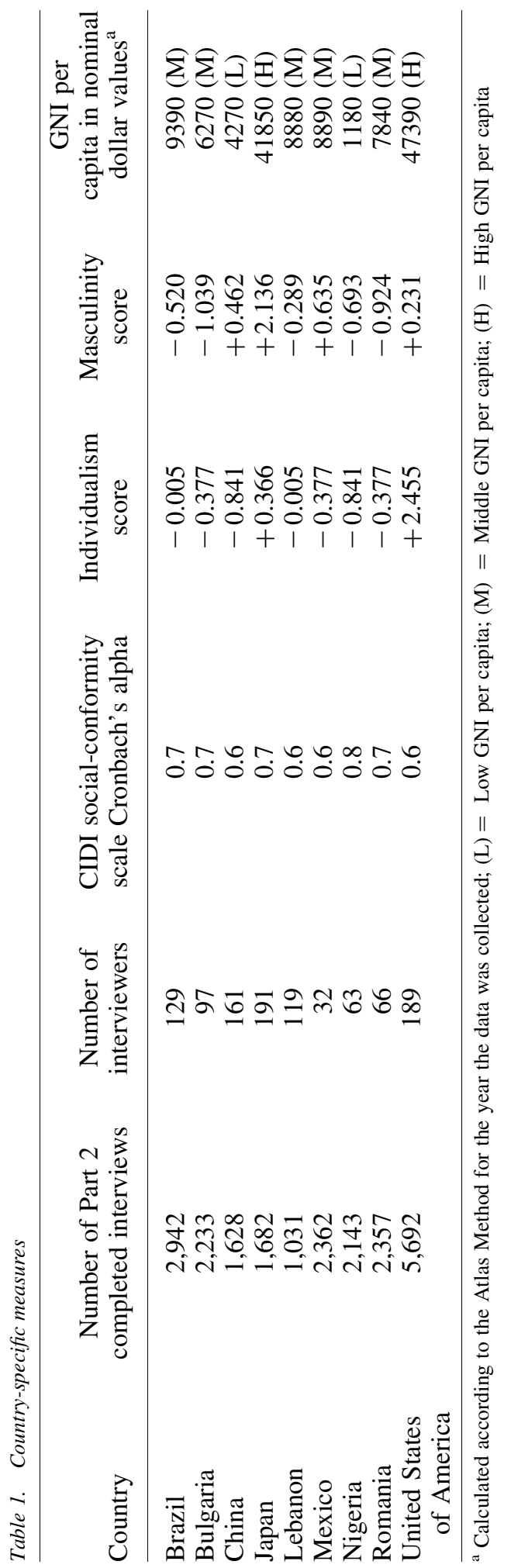


methods. Interviewer training in each country was modeled on a five-day training that project staff from each country had attended. All data were collected before 2008, with the majority of the fieldwork taking place between 2001 and 2003. Detailed information on the specific years of data collection, mode used, response rates, and supervisor-tointerviewer ratio are published elsewhere (Pennell et al. 2008).

\subsection{Measures Studied}

As detailed below in the analysis section, a multilevel model was used to explore our research questions. Below we list the key binary outcomes, followed by predictors.

\subsubsection{Key Outcomes}

The key outcome measures fell into three categories, representing a variety of sensitive behaviors, attitudes, and relatively neutral measures.

Behavioral outcome: CIDI 3.0 included a section on suicidality. In this section, respondents were asked to report whether they had ever made a suicide plan or attempted suicide. This outcome was chosen based on the authors' perceived judgment that reporting on suicidal behavior is relatively undesirable across cultures.

Attitudinal outcome: Married respondents were asked to rate their relationship with their current partner by answering the following question: "Using a scale from 0 to 10 where 0 means the worst possible marriage, and 10 means the best, how would you rate your marriage?" Since we were interested in investigating the effect of partner presence on reporting high marital ratings (rather than an average increase of one point on the scale), the score was categorized into two groups: a high rating defined as a score above the midpoint of the scale (which is five) and low rating (five or below). Reporting high marital rating is judged to be desirable if the partner is present.

Possible cultural variations in the sensitivity of reporting suicidal behavior and high marital rating were investigated through testing for a moderation effect of cultural background on third-party/partner presence.

Physical Chronic Condition Outcomes: Respondents were asked to report whether they had ever been told by any health professional that they had the following conditions: high blood pressure, asthma, or arthritis. These outcomes were chosen based on our judgment that such chronic conditions are less sensitive than suicidal behavior and marital rating across cultures.

A number of substantive and sociodemographic predictors were investigated. These predictors are described below.

\subsubsection{Respondent-Level Predictors}

Interview privacy: Interviewer observations about the privacy of the interview setting were collected at the end of the interview. Interviewers were instructed to specify: 1) whether a third person was present at any time during the interview; 2) the relationship of the third party to the respondent (parent, partner, child, youth, or other adults); and 3) the duration of the third party's presence during the interview (all the time, most of the time, about half of the time, about one quarter of the time, or less than one quarter of the time). The current analyses focus on any third-party presence, excluding children 
under the age of six (for the suicidal behavior outcome) and partner presence (for the marital rating outcome). The duration of the third-party stay was divided into two categories: 1) all of the interview time and 2) some of the interview time.

Respondent's need for social conformity: CIDI 3.0 included an adapted version of the Marlowe-Crowne Social Desirability Scale (Crowne and Marlowe 1960). Crowne and Marlowe designed the scale to measure the construct they labeled as "the need for social approval." The CIDI adapted scale consisted of 10 true or false statements such as "I never met a person that I didn't like" and "I have always told the truth." Respondents were instructed to choose the answer that first came to their mind and not take too much time thinking before they answered. A value of one was assigned to each item the respondent endorsed. The sum of these values formed a score ranging from zero to ten. Within each country, the respondent's total score was standardized by the country's average and standard deviation. A score was considered high if it was at least one standard deviation above the national-level mean. This measure will be referred to as the CIDI social-conformity scale, and was used to investigate whether an interaction between third-party presence and a high score on this scale had an impact on reporting sensitive information. Country-level Cronbach alpha estimates are in the acceptable to good range (0.6-0.8) (Table 1).

Respondent sociodemographic predictors: These variables were treated as control variables and included the respondent's gender, age (18-34, 35-49, 50-64, older than 64 years), marital status (never married, currently married or cohabiting, previously married), education level (high, middle, low, very low relative to the rest of the country), income (high, middle, low, very low relative to the rest of the country), current employment status (employed, studying, taking care of home, other), and household size (fewer than two, two, three, more than three). Among those currently married or cohabiting, the partner's level of education (high, middle, low, very low) and type of occupation (not employed, have a low-skill job, low-to-average skill job, average-to-high skill job, and high-skill job) were also taken into account.

\subsubsection{Interviewer-Level Predictors}

Interviewer identification numbers were available for all nine countries. This information enabled the modeling of random effects (intercepts) for the individual members of the interviewing force in each country. No other interviewer-level covariates were available.

\subsubsection{Country-Level Predictors}

Two country-level cultural dimensions were included in the current analyses: individualism and masculinity. Country-specific scores for each of the two dimensions and more details on their assessment are available in Hofstede et al. (2010). For each dimension and for each country, a standardized score was calculated based on the average score and the standard deviation across all the countries included in the analyses. Higher scores indicated higher levels of the underlying dimension. The country-specific scores are presented in Table 1.

Finally, the countries' economic strength and standard of living was measured by their Gross National Income (GNI) per capita. GNI measures in nominal dollar values that were calculated according to the Atlas Method for the year the data was collected were used. According to the World Bank, the Atlas method "applies a conversion factor that averages 
the exchange rate for a given year and the two preceding years, adjusted for differences in rates of inflation" ("GNI per capita," Atlas method (current US\$), accessed July 26, 2012, http://data.wordbank.org/indicator/NY.GNP.PCAP.CD). The limited number of countries and the vast differences in wealth leave big gaps in the GNI measure in this group of countries. The countries that are in this sample thus lend themselves to categories of wealth rather than a continuum of wealth (as evident in Table 1). Given these big gaps, the countries were categorized into three groups according to their GNI per capita: high, middle, and low GNI per capita. This categorization matches the World Bank classification of countries by income.

\section{Analysis}

Because of the limited number of countries, a two-level logistic regression with respondents nested within interviewers was used for all outcomes. Interviewers were treated as random effects. All predictors were treated as fixed effects, including the cultural factors. Indicator variables for individual countries were not included as fixed effects due to a resulting low-rank design matrix. Country effects were adjusted for through the three country-level variables - individualism, masculinity, and GNI per capita. The analyses were repeated using a three-level model with respondents at level 1, interviewers at level 2, and countries at level 3; the results with respect to our main hypotheses were consistent but less stable because of the limited number of countries in the analyses. Thus the analyses and the findings reported below are based on the two-level model.

For suicidal behaviors and physical chronic condition outcomes (high blood pressure, asthma, or arthritis), the main predictors included whether any third party was present during all of the interview time (vs. none of the time), any third party was present during some of the interview time (vs. none of the time), the respondent's level on the CIDI social-conformity scale (high vs. low), the country's level of individualism, and the country's level of GNI per capita (middle vs. high and low vs. high). Only cultural factors that have been demonstrated in the literature to be related to both interview privacy and social desirability were included as main predictors. The country's standardized masculinity score as well as respondent demographics and socioeconomic characteristics were also included in all the models as control variables. Interactions between third-party presence measures and a high score on the CIDI social-conformity scale, and between third-party presence measures and the country's level of individualism and wealth were tested. To maintain a parsimonious model, only significant interactions (with a $p$-value less than 0.05 ) were kept in the final model; nonsignificant interactions were removed.

The marital rating outcome was collected in only five out of the nine countries. Substantive predictors for the marital outcome rating included partner presence, the respondent's level on the CIDI social-conformity scale (high vs. low), and the country's level of GNI per capita (middle vs. high and low vs. high). The country's level of individualism was not included in the model as two of the five countries had the same level of individualism and there was not enough variation to explore. Respondent sociodemographic characteristics as well as the presence of any other third party (other than a partner) and country's level of masculinity were also included in all the models. Interactions between 
partner-presence measures and a high score on the CIDI social-conformity scale, and between partner-presence measures and the country's level of wealth were tested separately. To maintain a parsimonious model, only significant interactions (with a $p$-value less than 0.05 ) were kept in the final model; nonsignificant interactions were removed.

All multilevel models are unweighted. To explore the effect of weights on the findings, the analyses were replicated using weighted and unweighted single-level logistic regression models. The weights accounted for within-country differential probability of selection, postadjustment to the country's sociodemographic distributions, and subsampling to specific questionnaire sections. Adjusted and unadjusted point estimates were consistent (with singlelevel models being more stable), suggesting that the use of weights to adjust for informative sample design was not necessary for the relationships investigated. All analyses were conducted using the PROC GLIMMIX procedure in SAS version 9.2 (SAS institute, NC).

\section{Results}

\subsection{Outcome Rates and Interview Privacy Rates Across Countries}

Table 2 presents the different outcome rates in each of the countries included in the analyses. Weighted rates for combined suicide plan or suicide attempt reports differed greatly across countries, with the lowest rates (1.0\%) reported in Nigeria and Romania, and the highest rate $(7.5 \%)$ reported in the United States. High marital ratings were reported by the majority of respondents in all five countries where such ratings were collected. All rates were higher than $90 \%$.

All countries included questions on high blood pressure, asthma, and arthritis. Reported rates of asthma were generally low (mainly less than 5.5\%), except in the United States where $11.6 \%$ of the respondents reported having asthma. Arthritis and high blood pressure were more common and rates were more variable across countries. Arthritis rates range from $7.0 \%$ (Lebanon) to $33.3 \%$ (Romania) and high blood pressure rates range from $4.3 \%$ (Lebanon) to $24.1 \%$ (United States).

On average, $37 \%$ of the interviews were conducted in the presence of a third person. Table 3 presents the rates of third-party presence and duration of stay in each of the nine countries. In most countries, a third party was present for part of the interview rather than the entire interview (the average rate across countries was $26 \%$ ). On average, only $11 \%$ of the interviews had a third party present during all of the interview time. Partners were present in $19 \%$ of the interviews across all the countries. Again, partners were mostly present for some parts of the interview rather than the whole interview. Country-specific rates are presented in Table 4.

\subsection{Effect of Third-Party Presence on Reporting: Results from Multilevel Logistic Regression Model}

\subsubsection{Suicidal Behavior}

As hypothesized and shown in Table 5 (interaction model column), the respondent's need for social conformity moderated the relationship between third-party presence and 


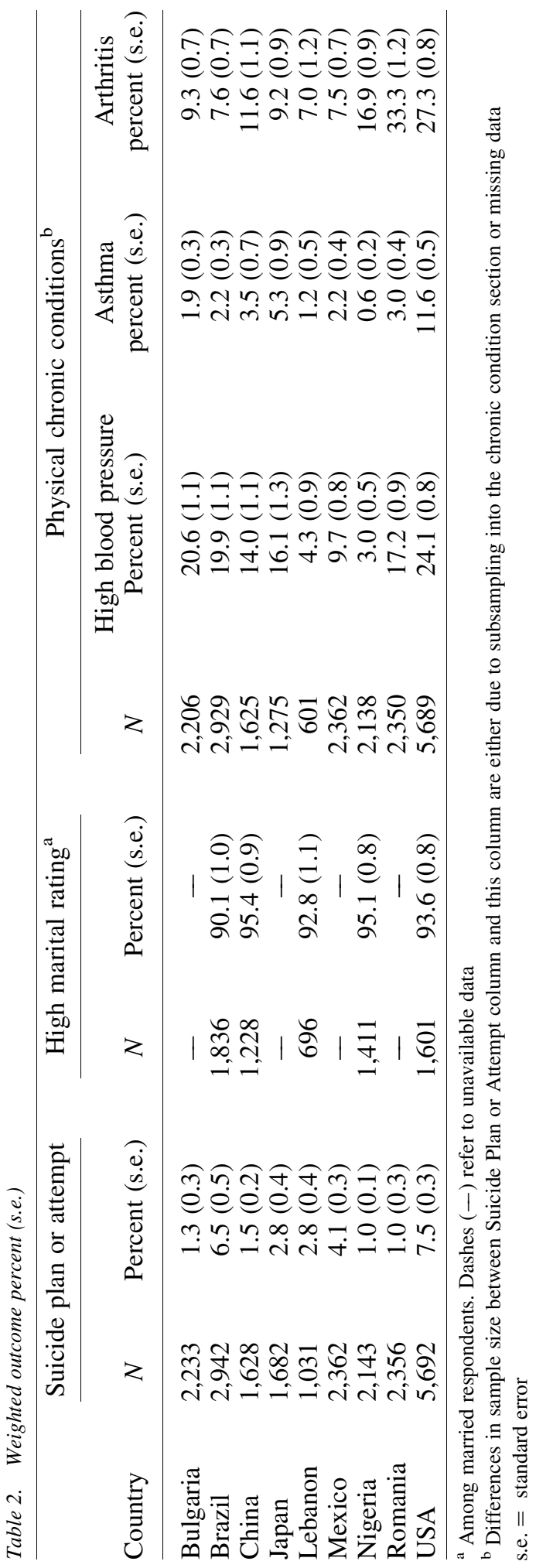


Table 3. Percentage of any third-party presence (s.e.)

\begin{tabular}{lcccc}
\hline Country & $N$ & $\begin{array}{c}\text { No third party } \\
\text { present during } \\
\text { interview }\end{array}$ & $\begin{array}{c}\text { Any third party } \\
\text { present all } \\
\text { interview time }\end{array}$ & $\begin{array}{c}\text { Any third party } \\
\text { present some } \\
\text { of the interview time }\end{array}$ \\
\hline Bulgaria & 2,232 & $60.7(1.0)$ & $13.2(0.7)$ & $26.1(0.9)$ \\
Brazil & 2,942 & $41.0(0.9)$ & $19.7(0.7)$ & $39.3(0.9)$ \\
China & 1,628 & $63.9(1.2)$ & $12.8(0.8)$ & $23.3(1.0)$ \\
Japan & 1,346 & $87.5(0.9)$ & $2.7(0.4)$ & $9.8(0.8)$ \\
Lebanon & 1,031 & $33.6(1.5)$ & $21.5(1.3)$ & $44.9(1.5)$ \\
Mexico & 2,350 & $65.4(1.0)$ & $10.7(0.6)$ & $23.9(0.9)$ \\
Nigeria & 2,141 & $68.0(1.0)$ & $7.1(0.6)$ & $24.9(0.9)$ \\
Romania & 2,356 & $64.6(1.0)$ & $15.2(0.7)$ & $20.2(0.8)$ \\
USA & 5,304 & $70.0(0.6)$ & $5.0(0.3)$ & $25.0(0.6)$ \\
\hline
\end{tabular}

Values are unweighted estimates of sample \% (standard error)

reporting suicidal behavior. Ignoring this interaction would give a misleading picture of the direction of third-party presence effects.

Respondents who scored high on the social-conformity scale and who had a third person present during the interview were less likely to report suicidal behavior compared to those who had no one present. Among respondents who scored high on social conformity, the odds of reporting suicidal behavior were lower by a factor of .92 when a third party was present during the entire interview and by a factor of .56 when a third party was present part of the time relative to not having a third party present during the interview (see Table 5). The results were quite different among respondents who scored low on the CIDI social-conformity scale. Among those respondents, having a third person present all or part of the time increased reporting of suicidal behavior compared to being interviewed alone $(\mathrm{OR}=1.20$ and 1.40 , respectively; see Table 5).

Contrary to what was hypothesized, however, neither the country's level of individualism nor its wealth significantly moderated the effect of third-party presence on reporting suicidal behavior.

Table 4. Percentage of partner presence (s.e.)

\begin{tabular}{lcccc}
\hline Country & $N$ & $\begin{array}{c}\text { No partner present } \\
\text { during the interview }\end{array}$ & $\begin{array}{c}\text { Partner present } \\
\text { all interview time }\end{array}$ & $\begin{array}{c}\text { Partner present some } \\
\text { of the interview time }\end{array}$ \\
\hline Bulgaria & 2,232 & $73.9(0.9)$ & $8.5(0.6)$ & $17.6(0.8)$ \\
Brazil & 2,942 & $73.3(0.8)$ & $9.6(0.5)$ & $17.1(0.7)$ \\
China & 1,628 & $80.1(1.0)$ & $7.1(0.6)$ & $12.8(0.8)$ \\
Japan & 1,346 & $93.8(0.7)$ & $1.5(0.3)$ & $4.7(0.6)$ \\
Lebanon & 1,031 & $68.3(1.5)$ & $11.1(1.0)$ & $20.6(1.3)$ \\
Mexico & 2,350 & $88.6(0.7)$ & $4.0(0.4)$ & $7.4(0.5)$ \\
Nigeria & 2,141 & $91.6(0.6)$ & $1.7(0.3)$ & $6.6(0.5)$ \\
Romania & 2,356 & $79.3(0.8)$ & $8.3(0.6)$ & $12.4(0.7)$ \\
USA & 5,304 & $82.9(0.5)$ & $2.9(0.2)$ & $14.2(0.5)$ \\
\hline
\end{tabular}

Values are unweighted estimates of sample \% (standard error) 
Table 5. Odds ratio and 95\% confidence interval from multilevel logistic model predicting suicide attempt or plan $(N=21,329)^{\mathrm{a}}$

\begin{tabular}{|c|c|c|}
\hline & Main model & Interaction model \\
\hline \multicolumn{3}{|l|}{ Presence of third party } \\
\hline Third party present all of the time & $1.20(0.99-1.43)$ & $1.20(0.98-1.46)$ \\
\hline Third party present some of the time & $1.31(1.16-1.45)$ & $1.40(1.23-1.60)$ \\
\hline No third party present & 1.00 & 1.00 \\
\hline \multicolumn{3}{|l|}{ Social-conformity score } \\
\hline High score ${ }^{b}$ & $0.69(0.59-0.82)$ & $0.82(0.67-1.01)$ \\
\hline Low score & 1.00 & 1.00 \\
\hline Individualism standardized score (IND) & $2.72(2.26-3.28)$ & $2.72(2.26-3.28)$ \\
\hline \multicolumn{3}{|l|}{ Country's GNI per capita } \\
\hline Low & $12.78(6.54-24.97)$ & $12.74(6.52-24.88)$ \\
\hline Middle & $12.49(6.96-22.41)$ & $12.45(6.94-22.34)$ \\
\hline High & 1.00 & 1.00 \\
\hline $\begin{array}{l}\text { Present all of the time } \times \\
\text { high social conformity }\end{array}$ & - & $0.92(0.55-1.54)$ \\
\hline $\begin{array}{l}\text { Present some of the time } \times \\
\text { high social conformity }\end{array}$ & - & $0.56(0.38-0.82)$ \\
\hline
\end{tabular}

\subsubsection{Marital Ratings}

High ratings of marital relationships were positively associated with partner presence during the interview, controlling for other respondent-level characteristics, the country's level of wealth and masculinity (see Main Model, Table 6). Unlike suicidal behavior (and contrary to what was hypothesized), the association between partner presence and reporting a high marital rating was not significantly moderated by the respondent's need for social conformity.

The effect of partner presence on reporting marital rating was, however, significant among respondents interviewed in a country with middle GNI per capita (compared to high GNI per capita, specifically the United States), as hypothesized. Respondents who were interviewed in a middle-income country and in the presence of their partner during the whole interview had more than 1.5 times the odds of reporting a high marital rating score compared to those who had no partner present and were in a country with high GNI per capita (see Interaction Model, Table 6). Though the interaction effect among respondents interviewed in low-income countries was in the hypothesized direction (and similar to middle-income countries), it was not statistically significant.

\subsubsection{Physical Chronic Conditions}

Unlike reports of suicidal behavior and marital relationship rating, reporting high blood pressure, asthma, or arthritis was not significantly associated with third-party presence (see Table 7). Moreover, as hypothesized, no significant interaction effects were found between third-party presence and the respondent's need for social conformity or with either of the country-level factors for any of the three outcomes. 
Table 6. Odds ratio and 95\% confidence interval from multilevel logistic model predicting high marital rating score $(N=6,595)^{\mathrm{a}}$

Main model

\begin{tabular}{|c|c|c|}
\hline \multicolumn{3}{|l|}{ Presence of partner } \\
\hline Partner present all of the time & $1.59(1.08-2.35)$ & $0.51(0.24-1.10)$ \\
\hline Partner present some of the time & $1.36(1.07-1.73)$ & $1.51(0.92-2.50)$ \\
\hline No partner present & 1.00 & 1.00 \\
\hline \multicolumn{3}{|l|}{ Social-conformity score } \\
\hline High score ${ }^{b}$ & $1.55(1.17-2.05)$ & $1.53(1.16-2.02)$ \\
\hline Low score & 1.00 & 1.00 \\
\hline \multicolumn{3}{|l|}{ GNI per capita } \\
\hline Low & $1.63(1.15-2.30)$ & $1.56(1.07-2.28)$ \\
\hline Middle & $0.70(0.47-1.03)$ & $0.66(0.43-1.01)$ \\
\hline High & 1.00 & 1.00 \\
\hline $\begin{array}{l}\text { Partner present all of the time } \times \\
\text { low GNI per capita }\end{array}$ & - & $1.35(0.92-1.98)$ \\
\hline $\begin{array}{l}\text { Partner present some of the time } \times \\
\text { low GNI per capita }\end{array}$ & - & $1.01(0.77-1.31)$ \\
\hline $\begin{array}{l}\text { Partner present all of the time } \times \\
\text { middle GNI per capita }\end{array}$ & - & $1.64(1.21-2.22)$ \\
\hline $\begin{array}{l}\text { Partner present some of the time } \times \\
\text { middle GNI per capita }\end{array}$ & - & $0.94(0.78-1.14)$ \\
\hline
\end{tabular}

\section{Discussion}

This article is the first to investigate whether the effect of third-party presence on reporting sensitive information is moderated by the respondent's need for social conformity and the respondent's country of residence. Three types of outcomes were investigated: a sensitive behavioral outcome (suicidal behavior), a sensitive attitudinal measure (marital rating), and three relatively neutral health measures (having high blood pressure, arthritis, or asthma). Third-party presence effects are moderated by the respondent's need for social conformity and the respondent's country of residence. Though such moderating effects were hypothesized for both outcomes, they differed depending on whether the outcome is behavioral or attitudinal.

For sensitive behaviors, specifically suicidal behavior, having a third party present during the interview was associated with lower odds of reporting such behavior only among respondents who had high scores on the social-conformity scale. Having someone present during the interview might create a contextual stimulus that strengthens the respondent's already existing need for social conformity and increases their perceived likelihood that revealing such behavior in the presence of a third party will trigger negative consequences. To avoid such consequences, respondents might prefer not to disclose such information in the presence of the third party, a phenomenon sometimes referred to as 


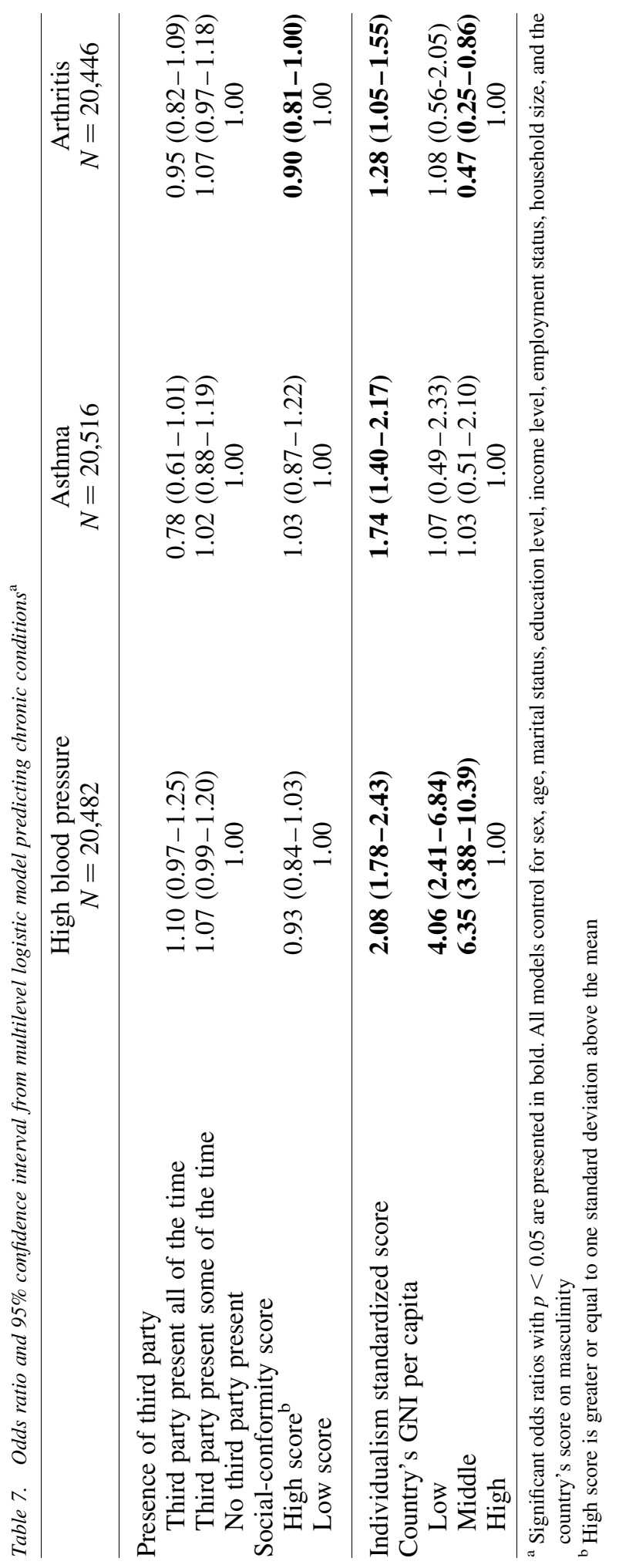


impression management. In fact, Paulhus (1984) shows that the Marlow Crowne Scale that is used in this paper as a measure of social conformity loads on impression management scales. The picture is quite different among respondents who do not have such needs and who scored low on social conformity. Among those respondents, the likelihood of reporting suicidal behavior was higher in the presence of a third party than in private. Respondents who scored low on social conformity might not have concerns about possible negative consequences from divulging such information, or they might have already confided in the third person present during the interview. Among such respondents, a third party who is present during the interview might act as a truth control increasing the reporting of such behavior. This has been also reported by two studies investigating sensitive undesirable outcomes in the United States (Aquilino 1997; Hoyt and Chaloupka 1994), where respondents who had a third party present were more likely to report illicit substance use than those interviewed in private. Future research on the effect of information already held by the third party on reporting sensitive information and how it interacts with respondent's need for social conformity is needed.

The interaction effect between the respondent's need for social conformity and thirdperson presence on reporting suicidal behavior was only statistically significant among interviews where a third person was present during "some" of the interview time. Though the direction of the interaction effect was the same when a third person was present "all" the interview time, it was not statistically significant. This difference between the two measures of third-party presence may reflect any of several factors: small cell size, psychological presence of third person and question location, and possible misclassification in the duration measure. First, the absence of a significant interaction effect between third person presence during "all" of the interview time and social conformity could be attributed to the small sample sizes, given that suicide behavior is rare (four percent in the overall sample) and that most of the nonprivate interviews had someone present "some" of the time rather than "all" of the time (70.4\% vs. $29.6 \%)$. Second, though it is not possible to ascertain that the third party was physically present during the specific administration of the suicide questions in the interviews where the third person was present during "some" of the interview time, we suspect that people are typically present during the beginning of the interview and they might leave or come and go as the interview progresses. This could have primed the respondent to perceive the setting as nonprivate, even after the third person had physically left the interview setting. In fact, there is strong evidence in the literature that social influence is not only produced by the actual physical presence of family and friends but also by their psychological presence, through priming respondents with their name, words or questions about them, or just thinking about or imagining them (Shah 2003; Moretti and Higgins 1999; Berscheid and Reis 1998; Fitzsimons and Bargh 2003). It seems that the activation of the representation of others occurs outside of people's awareness and has a powerful automatic effect on people's perceptions and behaviors (Berscheid and Reis 1998). Thus, given that the suicide behavior questions are towards the first third to the middle of the interview (depending on the skip patterns), even if the third person was not physically present during the actual administration of the suicide questions, his or her physical presence during earlier questions might have activated a mental representation of the third party's presence during the administration of the suicide questions. However, how long the psychological presence could persist during the 
interview is a question for future investigation. It is possible that the psychological presence would only last briefly after the third person left and thus would manifest only in nearby questions but not those administered towards the end of a long interview (such as the marital rating question). Third, the difference in the statistical significance between the two measures of third person presence could be also attributed to misclassification of the duration of presence in either directions. This could happen especially in situations where multiple people are present and come and go at different points during the course of the interview. In fact, $27 \%$ of interviews conducted in the presence of third members are reported to have more than one third person present.

Besides the respondent's need for social conformity, we hypothesized that the respondent's country of residence would moderate the effect of third-party presence on reporting suicidal behavior. However, this interaction effect was not significant. Although third-party presence is more common in collectivist and lower-income countries (Mneimneh 2012), the effect of third-party presence on reporting suicidal behavior does not change across countries that differ on those characteristics. It seems likely that reporting suicidal behavior is highly sensitive across all societies, irrespective of their level of collectivism/individualism or wealth. Even if the general level of social desirability is higher among collectivist and lower-income societies, having a third person present during the interview does not differentially heighten the sensitivity of suicidal information across countries.

The second sensitive outcome investigated is respondents' attitudes toward their current marriages. Respondents interviewed in the presence of their partner might be more motivated to provide a positive characterization of their relationship than those interviewed in private. This finding was first documented by Aquilino (1997). Unlike suicide behavior, the effect of partner presence on reporting marital attitudes was not moderated by the respondents' need for social conformity. The absence of the hypothesized moderating effect of the respondent's high need for social conformity on partner presence and reporting a high marital rating could be explained by the nature of the marital rating measure. Unlike suicidal behavior, which is a factual measure, marital rating is subjective. Respondents who scored low on social conformity might have already confided in other household members about their suicide experiences. Thus low conformity respondents might be more inclined to report their "true" suicidal behavior when a third party was present. This might not be the case for marital ratings, because marital happiness is not factual and does not have a "true" value.

When investigating whether the effect of partner presence on reporting high marital rating varies by the country of residence, we found it was significant in countries with middle GNI per capita (compared to high GNI per capita). Respondents interviewed in middle-income countries might practice impression management in the presence of their partner and deliberately report higher ratings of their marital relationship compared to their counterparts in high-income countries so as to maintain a favorable image in front of their spouses. Such reporting behavior (impression management) has been found to be more present among collectivist cultures (compared to individualistic cultures), which are typically less wealthy (Lalwani et al. 2006). Though such an effect did not reach statistical significance in low-income countries, the same trend is observed in low-income countries, and upon further investigation it was not statistically different from the effect in middle- 
income countries. It is also important to note that the magnitude of the interaction effect was higher before controlling for country's level of masculinity $(\mathrm{OR}=4.4 \mathrm{vs}$. $\mathrm{OR}=1.6$ after controlling for masculinity level). This could be attributed to the fact that the GNIper-capita interaction effect is partially driven by the country's level of masculinity.

As discussed earlier, differences in the observed interaction effects between countrylevel variables and interview privacy in reporting suicidal behavior versus marital ratings could be attributed to the difference between the two in levels of sensitivity. It is possible that suicidal behavior is sensitive across country boundaries. Thus, controlling for the respondent's level of social conformity and irrespective where he or she lives, the thirdparty presence decreases reporting suicidal behavior similarly since the topic is considered very sensitive across countries. This mechanism could be different for outcomes such as marital rating, however, where a differential level of sensitivity might be observed across countries, reflected through interactions between contextual interview settings such as partner presence and country-level variables such as wealth. The third set of outcomes investigated is chronic physical conditions: high blood pressure, arthritis, and asthma. These outcomes are normally perceived as more neutral than suicidal behavior or marital happiness. They were chosen to compare the effect of third-party presence on reporting clearly sensitive information with its effect on reporting more neutral outcomes. As hypothesized, the presence of a bystander during the interview does not significantly affect reporting of such neutral outcomes across the different cultures studied.

In summary, in our analysis of the World Mental Health data collected in a sample of countries, the presence of a third party during the interview affects reporting of sensitive outcomes. The effect can go in either direction, depending on the respondent's need for social conformity, the respondent's cultural setting, and the type of question. Whether it increases or reduces reporting of potentially sensitive information, the presence of a third party during the interview adds some measurement variation among respondents in a sample. Such variation is another layer of error that needs to be minimized. This is even more important in cross-cultural research, where both the rates of third-person presence (Mneimneh 2012) and their effects vary by culture, jeopardizing comparability.

Researchers sometimes try to counter the effect of third-party presence on reporting by using self-administered modes. Though the fact that using self-administered modes reduces interviewer effects has been established in the literature (Tourangeau and Yan 2007), whether such modes reduce the reporting effects of third-party presence is still under debate. Only five studies used randomized mode experiments and investigated the effect of third-party presence among each randomized group (Aquilino 1997; Aquilino et al. 2000; Cahucahrd 2013; Couper et al. 2003; Moskowitz 2004). The results of these studies are mixed. The presence of a third party during the interview, especially if the third party is a household member, might prime the respondent and alter his or her frame of mind when answering sensitive questions. Even when using a self-administered mode, the interview might not feel as private when a third party is present compared to when the respondent is interviewed alone. In fact, Aquilino et al. (2000) reported that third-party effects are found even when the bystander did not interfere with the interview or communicate with the respondent. Still, due to the limited empirical evidence, further research is needed on the moderating effect of the interview mode on third-party presence and reporting sensitive information before any conclusions are made. 
Given these findings, first, survey practitioners need to train interviewers better on how to achieve and maintain interview privacy, and the effect of such training interventions on interview privacy needs to be measured. Even when the mode of data collection uses selfadministration, third-party effects are found (Aquilino et al. 2000). Second, researchers are encouraged to measure and train interviewers on collecting more specific data on the interview setting, such as the timing of the presence through section-specific measures, its dynamics, and information already known by the third person to better understand the effect of third-party presence. Third, if such effects are replicated, researchers need to take these measures into consideration when analyzing their data and control for them in their substantive investigations.

The findings presented here need to be interpreted with the following considerations in mind. First, the presence of a third party at respondents' homes during the interview is difficult to control by the researcher and was not randomized. Though we controlled for many factors known to be associated with third-party presence, it is possible that there are other unmeasured factors that we did not control for and which could have affected the relationship observed between third-party presence and the reporting of sensitive outcomes. Thus no causal interpretations of any of the findings can be made and only associations are reported.

Second, interview privacy measures are based on interviewer observations reported at the end of the interview. The duration of third-party presence during the interview is an overall measure for the whole interview and is not section specific. Thus there could be some misclassification in the duration of the stay, especially in situations where multiple people might have been present at different points during the interview. Moreover, in instances where the interviewer reported that someone was present during "some" of the interview time, it was not possible to determine whether the bystander was actually present when the target question was asked. This could explain some of the differences observed between the effect of the third-party presence on reporting the different sensitive outcomes. Information on suicidal behavior was collected toward the first half of the interview and might have been affected by the "psychological" presence of a third person, whereas marital ratings were collected toward the end of the second half and might have been less prone to such a psychological presence in a long interview like the CIDI 3.0. The duration-of-stay measure also reflects all of the bystanders that might have been present during the interview. Thus, when investigating partner presence, if the interviewer indicated that another person was present in addition to the partner, the duration of stay was assigned to all of the different bystanders present. However, this should not significantly affect the findings as in the large majority of interviews (83\%), a partner was present but no other bystander was also present.

Third, the respondent's need for social conformity was measured using an adapted version of the Marlowe-Crowne Scale. Though this scale has been extensively used in the literature, and though the direction of the association between the respondent's need for social conformity and reporting outcomes is in the expected direction, these scales have their measurement problems. Self-reported scales that measure the need for social conformity are prone to misreporting, and their accuracy might vary depending on individual as well as cultural factors. Moreover, such scales assume that social conformity is stable from the time of its measurement; however, social conformity is contextual (Cialdini and Goldstein 2004) and could vary during the course of the interview. 
Fourth, suicidal behavior and the rating of marital-relationship outcomes were chosen based on the authors' judgment of their undesirability/desirability across cultures (compared to chronic conditions). Unfortunately, there is no empirical evidence of the level of sensitivity of these outcomes (in general or across cultures). We believe that suicidal behaviors are generally seen as undesirable in all cultures; similarly, rating one's current marital relationship highly (especially in the presence of one's partner) is generally desirable across all cultures. Cultural differences in the level of sensitivity of these outcomes were investigated by testing for the interaction effects, where larger third-party presence reporting effects were hypothesized for the cultures with higher levels of social desirability according to the literature.

Fifth, the cultural-dimension indices used in these analyses come from data published in Hofstede et al. (2010), some of which were collected several years ago. One concern is the applicability of those indices to the WMH data. Nevertheless, a number of researchers have shown that while the values of many nations have been changing, the relative positioning of those nations has been maintained (Hofstede et al. 2010; Ingelhart and Baker 2000; Schwartz et al. 2000).

Finally, the lack of statistical significance of some hypothesized interactions could be attributed to the small-size interaction classes resulting from the low prevalence of both sensitive outcomes and the specific privacy interview setting, namely the presence of a third party during "all" the interview time.

\section{Conclusion}

Reporting sensitive information is affected by the respondent's personal characteristics and cultural values, the social context in which the topic is broached, and the players involved during an interview. For a given topic, such factors affect whether the respondents interpret the content as socially desirable or undesirable and whether they edit the information or not. This article demonstrates that the effect of the privacy of the interview setting on reporting is moderated by the need for social conformity and respondent's country of residence.

It is important for us to develop a better understanding of the dynamics surrounding interview privacy, how it is affected by respondents' and third parties' personal characteristics and cultural background, and how privacy affects different types of survey questions. To achieve that, future work that captures what information is already held by the third party, as well as more specific interviewer privacy observations are needed. Such improved privacy measures need to then guide both practical interventions on training interviewers to better achieve, maintain, and observe interview privacy, and empirical work on the possible moderating effects of personal, cultural, and other interview-setting factors on the response process for sensitive questions.

\section{References}

Anderson, B.A. and B.D. Silver. 1987. "The Validity of Survey Response: Insights from Interviews of Married Couples in a Survey of Soviet Emigrants." Social Forces 66: 537-554. Doi: http://dx.doi.org/10.1093/sf/66.2.537. 
Aquilino, W. 1993. "Effects of Spouse Presence During the Interview on Survey Responses Concerning Marriage.” Public Opinion Quarterly 57: 358-376.

Aquilino, W. 1997. "Privacy on Self-Reported Drug Use: Interactions with Survey Mode and Respondent Characteristics." In The Validity of Self-Reported Drug Use: Improving the Accuracy of Survey Estimates (National Institute on Drug Abuse, Monograph 167), edited by L. Harrison and A. Hughes, pages 383-415. Rockville, MD: U.S. Department of Health and Human Services, National Institutes of Health, and National Institute on Drug Abuse, Division of Epidemiology and Prevention Research.

Aquilino, W.S., D.L. Wright, and A.J. Supple. 2000. "Response Effects Due to Bystander Presence in CASI and Paper-and-Pencil Surveys of Drug Use and Alcohol Use." Substance Use and Misuse 35: 845-867. Doi: http://dx.doi.org/10.3109/ 10826080009148424.

Bernardi, R.A. 2006. “Association Between Hofstede's Cultural Constructs and Social Desirability Response Bias.” Journal of Business Ethics 65: 43-53. Doi: http://dx.doi. org/10.1007/s10551-005-5353-0.

Berscheid, E. and H.T. Reis. 1998. "Attractions and Close Relationships." In The Handbook of Social Psychology, edited by D.T. Gilbert, S.T. Fiske, and L. Gardner, pages 193-281. New York: McGraw-Hill.

Bond, R. and P.B. Smith. 1996. "Culture and Conformity: A Meta-Analysis of Studies Using Asch's (1952b, 1956) Line Judgment Task." Psychological Bulletin 119: 111-137. Doi: http://dx.doi.org/10.1037/0033-2909.119.1.111.

Cialdini, R.B. and N.J. Goldstein. 2004. "Social Influence: Compliance and Conformity." Annual Review of Psychology 55: 591-621.

Casterline, J. and V.C. Chidambaram. 1984. "The Presence of Others During the Interview and the Reporting of Contraceptive Knowledge and Use." In Survey Analysis for the Guidance of Family Planning Programs, edited by J. A. Ross and R. McNamara, 267-298. Liege: Ordina Editions.

Cahucahrd, S. 2013. "Using MP3 Players in Surveys: The Impact of a Low-tech SelfAdministration Mode on Misreporting and Bystanders' Influence." Public Opinion Quarterly 77: 220-231. Doi: http://dx.doi.org/10.1093/poq/nfs060.

Couper, M.P., E. Singer, and R. Tourangeau. 2003. "Understanding the Effects of AudioCASI on Self-Reports of Sensitive Behavior.” Public Opinion Quarterly 67: 385-395.

Crowne, D.P. and D. Marlowe. 1960. “A New Scale of Social Desirability Independent of Psychopathology.” Journal of Consulting Psychology 24: 349-354. Doi: http://dx.doi. org/10.1037/h0047358.

Fitzsimons, G.M. and J.A. Bargh. 2003. "Thinking of You: Nonconscious Pursuit of Interpersonal Goals Associated with Relationship Partners." Journal of Personality and Social Psychology 84: 148-164. Doi: http://dx.doi.org/10.1037/0022-3514.84.1.148.

Gfroerer, J. 1985. "Influence of Privacy on Self-Reported Drug Use by Youths." In SelfReport Methods of Estimating Drug Use: Meeting Current Challenges to Validity, edited by B.A. Rouse, N.J. Kozel, and L.G. Richards, pages 22-30. Rockville, MD: U.S. Department of Health and Human Services, Public Health Service, Alcohol, Drug Abuse, and Mental Health Administration, and National Institute on Drug Abuse.

Harkness, J., B. Pennell, A. Villar, N. Gebler, S. Auilar-Gaxiola, and I. Bilgen. 2008. "Translation Procedures and Translation Assessment in the World Mental Health 
Survey Initiative." In The WHO World Mental Health Surveys: Global Perspectives on the Epidemiology of Mental Disorders, edited by R.C. Kessler and T.B. Üstün, pages 91-113. New York: Cambridge University Press.

Heeringa, S.G., J.E. Wells, F. Hubbard, Z.N. Mneimneh, W. Chiu, N.A. Sampson, and P.A. Berglund. 2008. "Sample Designs and Sampling Procedures." In The WHO World Mental Health Surveys: Global Perspectives on the Epidemiology of Mental Disorders, edited by R.C. Kessler and T.B. Üstün, pages 14-32. New York, NY: Cambridge University Press.

Hofstede, G., G.J. Hofstede, and M. Minkov. 2010. Culture and Organizations: Software of the Mind, 3rd ed. New York, NY: McGraw-Hill.

Hoyt, G.M. and F.J. Chaloupka. 1994. "Effect of Survey Conditions on Self-Reported Substance Use.” Contemporary Economic Policy 7: 109-121. Doi: http://dx.doi.org/10. 1111/j.1465-7287.1994.tb00439.x.

Ingelhart, R. and W.E. Baker. 2000. "Modernization, Cultural Change, and the Persistence of Traditional Values." American Sociological Review 65: 19-51.

Johnson, T.P. and F.J.R. van de Vijver. 2003. "Social Desirability in Cross-Cultural Research.” In Cross-Cultural Survey Methods, edited by J.A. Harkness, F.J.R. van de Vijer, and P.P. Mohler, pages 195-206. Hoboken, NJ: Wiley.

Kessler, R.C., J. Abelson, O. Demler, J.I. Escobar, M. Gibbon, M.E. Guyer, M.J. Howes, R. Jin, W.A., Vega, E.E. Walters, P. Wang, A. Zaslavsky, and H. Zheng. 2004. "Clinical Calibration of DSM-IV Diagnoses in the World Mental Health (WMH) Version of the World Health Organization (WHO) Composite International Diagnostic Interview (CIDI)." International Journal of Methods in Psychiatric Research 13: 122-139. Doi: http://dx.doi.org/10.1002/mpr.169.

Kessler, R.C. and T.B. Üstün. 2004. "The World Mental Health (WMH) Survey Initiative version of the World Health Organization (WHO) Composite International Diagnostic Interview (CIDI)." International Journal of Methods in Psychiatric Research 13: 93-121.

Lalwani, A.K., S. Shavitt, and T. Johnson. 2006. "What is the Relation Between Cultural Orientation and Socially Desirable Responding?" Journal of Personality and Social Psychology 90: 165-178. Doi: http://dx.doi.org/10.1037/0022-3514.90.1.165.

Moretti, M.M. and E.T. Higgins. 1999. "Internal Representations of Others in SelfRegulations: A New Look at a Classic Issue.” Social Cognition 17: 186-208. Doi: http://dx.doi.org/10.1521/soco.1999.17.2.186.

Mneimneh, Z.N. 2012. "Interview Privacy and Social Conformity Effects on Socially Desirable Reporting Behavior: Importance of Cultural, Individual, Question Design and Implementation Factors." Available at: http://deepblue.lib.umich.edu/handle/2027. 42/96051

Moskowitz, J.M. 2004. “Assessment of Cigarette Smoking and Smoking Susceptibility Among Youth: Telephone Computer-Assisted Self-Interviews Versus ComputerAssisted Telephone Interviews." Public Opinion Quarterly 68: 565-587. Doi: http://dx. doi.org/10.1093/poq/nfh040.

Paulhus, D.L. 1984. "Two-Component Models for Socially Desirable Responding." Journal of Personality and Social Psychology 46: 598-609. Doi: http://dx.doi.org/10. 1037/0022-3514.46.3.598. 
Pennell, B., Z.N. Mneimneh, A. Bowers, S. Chardoul, J.E. Wells, M.C. Viana, K. Dinkelmann, N. Gebler, S. Florescu, Y. He, Y. Huang, T. Toma, and G.V. Saiz. 2008. "Implementation of the World Mental Health Surveys." In The WHO World Mental Health Surveys: Global Perspectives on the Epidemiology of Mental Disorders, edited by R.C. Kessler and T.B. Üstün, pages 33-57. New York: Cambridge University Press. Pennell, B., J.A. Harkness, R. Levenstein, and M. Quaglia. 2010. "Challenges in CrossNational Data Collection." In Survey Methods in Multinational, Multiregional, Multicultural Contexts, edited by J.A. Harkness, M. Braun, B. Edwards, T.P. Johnson, L. Lyberg, P. Mohler, B. Pennell, and T.W. Smith, pages 269-298. Hoboken, NJ: John Wiley \& Sons.

Podmore, D., D. Chaney, and P. Golder. 1975. "Third Parties in the Interview Situation: Evidence from Hong Kong." The Journal of Social Psychology 95: 227-231. Doi: http://dx.doi.org/10.1080/00224545.1975.9918708.

Pollner, M. and R.E. Adams. 1994. "The Interpersonal Context of Mental Health Interviews." Journal of Health and Social Behavior 35: 283-290.

Pollner, M. and R.E. Adams. 1997. "The Effect of Spouse Presence on Appraisals of Emotional Support and Household Strain.” Public Opinion Quarterly 61: 615-626. Doi: http://dx.doi.org/10.1086/297820.

Schwartz, S.H., A. Bardi, and G. Bianchi. 2000. "Value Adaptation to the Imposition and Collapse of Communist Regimes in East-central Europe." In Political Psychology: Cultural and Cross-cultural Foundations, edited by S.A. Renshon and J. Duckitt, pages 217-237. London: Macmillan.

Shah, J. 2003. "Automatic for the People: How Representations of Significant Others Implicitly Affect Goal Pursuit." Journal of Personality and Social Psychology 84: 661-681. Doi: http://dx.doi.org/10.1037/0022-3514.84.4.661.

Smith, P.B., M.H. Bond, and Ç. Kağıtçıbaşı. 2006. Understanding Social Psychology Across Cultures: Living and Working in a Changing World. London: Sage Publications. Smith, T.W. 1997. "The Impact of the Presence of Others on a Respondent's Answers to Questions.” International Journal of Public Opinion Research 9: 33-47. Doi: http://dx. doi.org/10.1093/ijpor/9.1.33.

The World Bank, "GNI per Capita, Atlas Method (current US\$)" Accessed July 26, 2012. Available at: http://data.worldbank.org/indicator/NY.GNP.PCAP.CD.

Ting-Toomey, S. 1999. Communicating Across Cultures. New York: Guilford Press.

Tourangeau, R. and T. Yan. 2007. "Sensitive Questions in Surveys." Psychological Bulletin 133: 859-883. Doi: http://dx.doi.org/10.1037/0033-2909.133.5.859.

Triandis, H.C. 1989. "The Self and Social Behaviour in Differing Cultural Contexts." Psychological Review 96: 506-520. Doi: http://dx.doi.org/10.1037/0033-295X.96. 3.506 .

Triandis, H.C. 1995. Individualism and Collectivism. Boulder, CO: Westview Press.

Van de Vijver, F.J.R. 2003. "Bias and Equivalence: Cross-Cultural Perspectives." In Cross-cultural Survey Methods, edited by J.A. Harkness, F.J. R. van de Vijver, and P.P. Mohler, pages 143-156. Hoboken, NJ: Wiley.

Van Hemert, D.A., F.J.R. van de Vijver, Y.H. Poortinga, and J. Georgas. 2002. "Structural and Functional Equivalence of the Eysenck Personality Questionnaire Within and 
Between Countries." Personality and Individual Differences 33: 1229-1249. Doi: http://dx.doi.org/10.1016/S0191-8869(02)00007-7.

Welkenhuysen-Gybels, J. and J. Billiet. 2001. "The Impact of Third Party Presence in Survey Interviews on the Measurement of Political Knowledge." Acta Politica 36: 287-306.

Received June 2013

Revised May 2015

Accepted June 2015 